Research Article

\title{
Robust Cross-Orthogonality Check Using the Principle of Local Correspondence
}

\author{
Anders Skafte $\mathbb{D}^{\mathrm{D}},{ }^{1}$ Manuel Aenlle Lopez $\mathbb{D}^{2},{ }^{2}$ and Rune Brincker ${ }^{3}$ \\ ${ }^{1}$ Vestas A/S, Hedager 42, Aarhus N, 8200, Denmark \\ ${ }^{2}$ Department of Construction and Manufacturing Engineering, University of Oviedo, Gijón, Spain \\ ${ }^{3}$ Center for Oil and Gas, Technical University of Denmark, Lyngby, Denmark
}

Correspondence should be addressed to Anders Skafte; mail@andersskafte.dk

Received 26 September 2019; Revised 4 August 2020; Accepted 5 September 2020; Published 17 September 2020

Academic Editor: Jean-Mathieu Mencik

Copyright (C) 2020 Anders Skafte et al. This is an open access article distributed under the Creative Commons Attribution License, which permits unrestricted use, distribution, and reproduction in any medium, provided the original work is properly cited.

The cross-orthogonality check (XOR) is a widely used correlation measure for validating finite element (FE) models, where the orthogonality between analytical and experimental mode shapes is measured as the inner product over the mass matrix. Ideally, this yields the identity matrix where any deviation from this matrix can be seen as a lack of correlation. One of the drawbacks of this measure is its sensitivity to noise on the experimental mode shapes, which can have a significant influence. The present paper presents a new way of calculating the XOR which provides robust results towards noise. The method, known as the principle of local correspondence (LC), is a mode shape-based technique for expanding experimental mode shapes using a unique linear combination of FE modes. The advantage of using the LC principle for calculating the XOR is that no reduced mass matrix is needed, and the influence towards noise on the mode shapes is reduced compared with other known techniques. In this paper, the method is validated using probabilistic numerical investigations. An FE model of a shell structure is used as a case study where Monte Carlo simulations are used to change the material properties and create a variety of different noise scenarios. The results are compared with similar simulations using Guyan and SEREP.

\section{Introduction}

Finite element (FE) models are some of the most important tools for engineers working with advanced civil structures subjected to dynamic loading. The FE models provide valuable information in the design phase where a wide variety of structural designs, materials, and advanced load scenarios can be tested. Once the structure is built, it might be necessary to measure the correlation between the experimental results and the analytical model to validate the analysis made with the FE model. When focusing on the dynamic properties of the structure, the correlation can be determined using a variety of different measures such as the modal assurance criterion (MAC), the coordinate modal assurance criterion (COMAC), the frequency response assurance criterion (FRAC), and more [1]. The cross-orthogonality check (XOR) is popular as a correlation measure when comparing analytical and experimental mode shapes.
Here, the orthogonality between the experimental and analytical mode shapes is measured as the inner product over the mass matrix. Ideally, this yields the identity matrix, whereas any off-diagonal element can be seen as a coupling of modes. The acceptable limits for the XOR are often dictated by large agencies such as NASA, United States Air Force, and ESA [2-4]. These limits vary, but often diagonal terms above 0.95 and off-diagonal terms below 0.1 are considered acceptable.

The XOR cannot be calculated directly since the mode shapes found from experimental tests only include a limited amount of degrees of freedom (DOF) compared with the mode shapes from the FE model. This leaves two options: the experimental mode shapes must be expanded, so they include as many DOFs as the mode shapes from the FE model, or the FE mass matrix must be reduced, the latter referred to as a test analysis model (TAM). A reduced version of the mass matrix can be estimated using reduction techniques 
such as Guyan [5], dynamic reduction [6], improved reduced system (IRS) [7], system equivalent reduction expansion process (SEREP) [8], and the modal TAM [9].

There are, however, some problems regarding the XOR. The results will be highly dependent on the amount of noise that always will be present when identifying experimental mode shapes, as well as the precision in which the TAM has been calculated. This can lead to off-diagonal terms which make it hard to fulfill the requirements for an acceptable correlation. Further studies show that the existence of closely spaced modes can lead to large coupling terms due to the high sensitivity of the mode shapes [10].

Several other authors have analyzed the sensitivity of the XOR using a probabilistic approach. In [11], an alternative to the XOR is formulated. This correlation measure, named XORviaGDOP, is based on the description of general projection and has the advantage that it does not require a reduced mass matrix. In [12], this method is used to develop an uncertainty index relating to the XOR. The method is validated by a probabilistic investigation of an analytical model of a cantilever beam. In [13], a stochastic framework for analyzing the sensitivity of the XOR due to noise on the mode shapes is presented. An FE model of a satellite is used as a case study, where $2 \%$ noise is added to the mode shapes to simulate an experiment, and the article focuses on how the optimal sensor placement for individual TAMs performs. In [14], the author also uses an FE model of a satellite as a test case. Here, the focus is primarily on the number of FE modes used as a basis in SEREP as well as different noise levels on the mode shapes. By using Monte Carlo simulation, the paper presents how the size of the subset of FE modes as well as the amount of noise of the mode shapes affect the quality of the XOR. Similar studies on the robust of the XOR can be found in papers such as $[15,16]$.

In this paper, it is proposed that the XOR should be calculated using a projection of a unique cluster of FE modes onto each experimental mode, following the principles of local correspondence (LC) [17]. The LC principle was originally formed as an expansion method, and like SEREP, this method uses a combination of FE modes to expand the experimental modes. However, the LC principle states that only a cluster of FE modes surrounding the given experimental mode should be used in the expansion.

The theory presented in this paper shows that the matrix describing the linear combination between experimental modes and analytical modes is equivalent to the XOR, which has the advantage that the XOR can be calculated without either expanding the mode shapes or reducing the mass matrix.

This technique is validated using a probabilistic approach where the main focus is on how noise on the experimental mode shapes affects the diagonal and offdiagonal elements when calculating the XOR. An FE model of a shell structure is used as case study, where noise is added to the mode shapes to simulate modes extracted from experiments. By using Monte Carlo simulation, the distribution of diagonal and off-diagonal terms can be investigated for a wide variety of noise scenarios and material properties. The proposed method is compared with similar analysis using SEREP and Guyan.

\section{Background Theory}

When FE mode shapes are scaled to mass unity, the definition of orthogonality between the modes with respect to the mass matrix is given by

$$
\mathbf{B}^{T} \mathbf{M B}=\mathbf{I},
$$

where $\mathbf{B}$ is the mass-scaled FE mode shapes, $\mathbf{M}$ is the FE mass matrix, and I denotes the identity matrix. The XOR is then calculated by substituting the left FE mode shape matrix with the mode shapes from the experiment, and the full mass matrix with a TAM:

$$
\mathbf{X O R}_{\mathrm{red}}=\mathbf{A}_{a}^{T} \mathbf{M}_{T A M} \mathbf{B}_{a},
$$

where A denotes the experimental mode shapes. The subscript $a$ refers to a truncated mode shape matrix containing only the active DOFs and subscript red refers to the XOR has been calculated using a reduced mass matrix.

However, the XOR can also be calculated by expanding the experimental mode shapes and using the full FE mass matrix:

$$
\mathbf{X O R}_{\text {full }}=\widehat{A}^{T} \mathbf{M B},
$$

with $\widehat{A}$ indicating an estimate, in this case, the expanded experimental mode shapes, and subscript full refers to the XOR has been calculated using the full mode shape matrix.

Ideally, both equations (2) and (3) yield the identity matrix meaning that the experimental and FE mode shapes are identical. This will, though, never be the case due to reasons such as noise on the experimental mode shapes, errors in calculating the TAM or discrepancies between the analytical and experimental model. To validate if the FE model is well correlated with the experimental mode shapes, the diagonal and off-diagonal elements in the XOR have to fulfill certain threshold values here chosen as

$$
\begin{aligned}
& \mathrm{XOR}_{i, j}>0.95 \text { for } i=j, \\
& \mathrm{XOR}_{i, j}<0.1 \text { for } i \neq j .
\end{aligned}
$$

The subscripts $i$ and $j$ indicate row and column number.

Likewise, the threshold for acceptable correlation between natural frequencies can be determined by [2-4]

$$
\Delta f_{i}=\frac{\left|f_{A, i}-f_{B, i}\right|}{f_{B, i}} 100 \%,
$$

with $f_{A, i}$ and $f_{B, i}$ being the $i$-th natural frequency for the experiment and FE models, respectively. The limit for $\Delta f_{i}$ varies for different agencies but also depends on the frequency bands in which each mode is present. A deviation of $3-5 \%$ is often considered acceptable.

2.1. Guyan Reduction. If equation (2) is used to find the XOR, a TAM must be created. Equal for many of the wellknown reduction techniques is that the mass matrix can be reduced by 


$$
\mathbf{M}_{\mathrm{TAM}}=\mathbf{T}^{T} \mathbf{M T},
$$

with $\mathbf{T}$ being a matrix describing the transformation between the active and full set of DOFs in the FE model. The definition of $\mathbf{T}$ is what defines the different reduction techniques.

One of the first reduction techniques invented was the Guyan reduction [5] which is one of the techniques most frequently seen implemented in commercial software. The method has its origin in the equation of static equilibrium. The DOFs in the FE model are divided into two groups: active and deleted:

$$
\left[\begin{array}{ll}
\mathbf{K}_{a a} & \mathbf{K}_{a d} \\
\mathbf{K}_{d a} & \mathbf{K}_{d d}
\end{array}\right]\left\{\begin{array}{l}
\mathbf{x}_{a} \\
\mathbf{x}_{d}
\end{array}\right\}=\left\{\begin{array}{c}
\mathbf{f}_{a} \\
0
\end{array}\right\},
$$

with $\mathbf{K}$ being the stiffness matrix and $\mathbf{f}_{a}$ being the force acting on the active DOFs.

In equation (7), it is assumed that the force is only acting on the active DOFs. The lower part of equation (7) gives the relation between the active and deleted DOFs which can be expressed by a transformation matrix:

$$
\mathbf{T}_{G}=\left[\begin{array}{c}
\mathbf{I} \\
-\mathbf{K}_{d d}^{-1} \mathbf{K}_{a d}
\end{array}\right],
$$

with the subscript $\mathbf{G}$ indicating that the transformation matrix is found using Guyan. A TAM can then be calculated using equation (6).

2.2. SEREP. SEREP [8] was originally invented as an expansion technique but is just as often used for reduction. What separates SEREP from many of the other reduction/ expansion methods is that the process is revisable, meaning that a set of mode shapes can be reduced and expanded without any loss of precision.

Considering the equation of motion for an undamped linear system,

$$
\mathbf{M} \ddot{x}+\mathbf{K} \mathbf{x}=0 .
$$

The solution can be described as a linear combination of the system's mode shapes and its modal coordinates. Once again dividing the DOFs into active and deleted DOFs yields

$$
\mathbf{x}=\left\{\begin{array}{l}
\mathbf{x}_{a} \\
\mathbf{x}_{d}
\end{array}\right\}=\left[\begin{array}{l}
\mathbf{B}_{a} \\
\mathbf{B}_{d}
\end{array}\right] \mathbf{q}
$$

with $\mathbf{q}$ being the modal coordinates.

Using the upper part of equation (10) and assuming that the number of active DOFs is larger than the number of modes in the mode shape matrix, an estimate of the modal coordinates can be found by

$$
\mathbf{q}=\mathbf{B}_{a}^{\dagger} \mathbf{x}_{a},
$$

with $\dagger$ indicating the Moore-Penrose pseudoinverse.

The relation between the full and reduced systems is then found by substituting the expression for the modal coordinates, found in equation (11) into equation (10), which gives the transformation matrix:

$$
\mathbf{T}_{s}=\mathbf{B B}_{a}^{\dagger} .
$$
(6).

And again, the reduced mass matrix is found by equation

\section{The Principle of Local Correspondence}

3.1. Background. The principle of local correspondence (LC) [17] is a mode shape-based expansion technique which utilizes the fact that a change in a mode shape primarily is described as a linear combination of a cluster of the surrounding modes. The LC principle follows the same basic equations as SEREP, but the deduction follows another path having its origin in the mode shape sensitivity equations $[18,19]$.

Let us assume that a structure and its corresponding FE model are well correlated, so only small changes occur. An experimental mode shape can then be described as the sum of the corresponding FE mode and a change in the FE mode:

$$
\mathbf{a}_{i}=\mathbf{b}_{i}+\Delta \mathbf{b}_{i} .
$$

Heylen et al. [19], following the ideas by Nelson [18] about the theory of sensitivity analysis and thus also the theory of mode shape sensitivity, showed that the derivative of a given mode shape with respect to a perturbation in the model given by the parameter $u$ is expressed as

$$
\frac{\partial \mathbf{b}_{i}}{\partial u}=-\frac{1}{2 m_{i}} \mathbf{b}_{i}^{T} \frac{\partial \mathbf{M}}{\partial u} b_{i} b_{i}+\sum_{r=1, r \neq i}^{N} \frac{1}{\omega_{i}^{2}-\omega_{r}^{2}} \frac{1}{m_{r}} \mathbf{b}_{r}^{T}\left(-\omega_{i}^{2} \frac{\partial \mathbf{M}}{\partial u}+\frac{\partial \mathbf{K}}{\partial u}\right) \mathbf{b}_{i} \mathbf{b}_{r},
$$

where $m$ is the modal mass, $N$ is the total number of modes, and $\omega$ is the angular frequency.

Assuming that the perturbation is caused by a finite, but small, change in the mass matrix, equation (14) can be rewritten:

$$
\Delta \mathbf{b}_{i} \cong-\frac{1}{2 m_{i}} \mathbf{b}_{i}^{T} \Delta \mathbf{M} \mathbf{b}_{i} \mathbf{b}_{i}-\frac{1}{m_{r}} \sum_{r=1, r \neq i}^{N} \frac{1}{\omega_{i}^{2}-\omega_{r}^{2}}\left(\omega_{i}^{2} \mathbf{b}_{r}^{T} \Delta \mathbf{M} \mathbf{b}_{i}-\mathbf{b}_{r}^{T} \Delta K \mathbf{b}_{i}\right) \mathbf{b}_{r} .
$$

If the modes are scaled to mass unity, the modal masses, i.e., the terms $m_{i}=1$ and $m_{r}=1$, and utilizing that the terms $p_{i}=\mathbf{b}_{i}^{T} \Delta \mathbf{M} \mathbf{b}_{i}$ and $p_{r}=\left(1 / \omega_{i}^{2}-\omega_{r}^{2}\right)\left(\omega_{i}^{2} \mathbf{b}_{r}^{T} \Delta \mathbf{M} \mathbf{b}_{i}-\mathbf{b}_{r}^{T} \Delta \mathbf{K} \mathbf{b}_{i}\right)$ are both inner products, (15) can be simplified:

$$
\Delta \mathbf{b}_{i} \cong \mathbf{b}_{i} p_{i}-\sum_{r=1, r \neq i}^{M} \mathbf{b}_{r} p_{r},
$$

with $p_{i}$ and $p_{r}$ describing unknown scalars.

Inserting this expression into equation (13) and changing to a matrix formulation, the expression for the experimental mode shape can be simplified to

$$
\mathbf{a}_{i} \cong \mathbf{B} \mathbf{p}_{i}
$$

where the transformation vector $\mathbf{p}_{i}$ defines the linear combination of FE modes that describes the $i$-th experimental mode. A more thorough derivation of this theory is found in [17]. 
3.2. Choosing an Optimal Cluster of FE Modes. Where equation (13) to equation (17) cover the theoretical explanation of the LC principle, an iterative procedure must be used when determining the optimal subset of FE modes to expand the individual experimental mode shapes. An estimate of the projection matrix $\widehat{\mathbf{p}}_{i}$ can be found using classical least squares:

$$
\widehat{p}_{i}=\mathbf{B}_{a, i}^{\dagger} \mathbf{a}_{a, i},
$$

with $\mathbf{B}_{a, i}$ being the specific subset of FE mode best suited for expanding the $i$-th experimental mode.

And the experimental mode can be expanded by multiplying the subset of FE modes with the transformation vector:

$$
\widehat{a}_{i}=\mathbf{B}_{i} \widehat{p}_{i}
$$

where $\mathbf{B}_{i}$ can contain all the DOFs in the FE model.

However, problems can arise when the mode-to-DOF ratio becomes too large and overfitting can occur. By evaluating equation (15), it is clear that the mode shapes near to the considered mode in terms of frequency will have the largest influence when calculating $\Delta \mathbf{b}_{i}$. When expanding the experimental mode $\mathbf{a}_{i}$, the first step is to rearrange the order of the mode shapes in $\mathbf{B}_{a, i}$, so the first mode will be the one that correlates the best with $\mathbf{a}_{i}$, and the rest follows according to the distance in frequency in descending order. The next step is then iteratively to calculate transformation vector $\mathbf{p}_{i}$ equation (18) and expanded experimental mode shape equation (20), increasing the number of FE modes for each iteration. The subset of FE modes that gives the best correlation between $\mathbf{a}_{i}$ and $\widehat{a}_{i}$ is chosen to calculate $\mathbf{p}_{i}$.

A more thorough deduction of this proof can be found in [17]. A similar method is described in [20].

\section{Mode Shape-Based XOR}

When calculating the XOR using classic methods such as Guyan, dynamic reduction, or IRS, there is always a need for either reducing the mass matrix or expanding the experimental mode shapes. When using both SEREP [21] and LC, the XOR can be calculated using only the mode shapes from the two models.

4.1. XOR Using SEREP. SEREP is a reversible process meaning that reduction and expansion can be performed without any loss of precision. This also means that calculating the XOR with SEREP using either a reduced mass matrix or expanded mode shapes will give the same result.

The experimental mode shapes can be expanded by premultiplying these with the transformation matrix:

$$
\widehat{A}=\mathbf{T}_{S} \mathbf{A}_{a} \text {. }
$$

Inserting this expression into equation (3) and using equation (12) yields the following expression for the XOR using the full mass matrix:

$$
\mathbf{X O R}_{\text {full }}=\mathbf{A}_{a}^{T}\left(\mathbf{B}_{a}^{\dagger}\right)^{T} \mathbf{B}^{T} \mathbf{M B}
$$

This can be reduced due to the orthogonality conditions described in equation (1):

$$
\mathrm{XOR}_{\text {full }}=\mathbf{A}_{a}^{T}\left(\mathbf{B}_{a}^{\dagger}\right)^{T} .
$$

On the other hand, using equation (2) to calculate the $\mathrm{XOR}$ requires the reduced mass matrix, which in terms of SEREP can be found by using equations (6) and (12) (see also [21]):

$$
\mathbf{M}_{S}=\left(\mathbf{B}_{a}^{\dagger}\right)^{T} \mathbf{B}_{a}^{\dagger}
$$

Applying the expression for $\mathbf{M}_{S}$ into equation (2) gives

$$
\mathbf{X O R}_{\mathrm{red}}=\mathbf{A}_{a}^{T}\left(\mathbf{B}_{a}^{\dagger}\right)^{T} \mathbf{B}_{a}^{\dagger} \mathbf{B}_{a} .
$$

Now, substituting the definition of the Moore-Penrose pseudoinverse into equation (24),

$$
\mathbf{X O R}_{\mathrm{red}}=\mathbf{A}_{a}^{T}\left(\mathbf{B}_{a}^{\dagger}\right)^{T}\left(\mathbf{B}_{a}^{T} \mathbf{B}_{a}\right)^{-1} \mathbf{B}_{a}^{T} \mathbf{B}_{\mathrm{a}},
$$

which can be reduced to

$$
\mathbf{X O R}_{\mathrm{red}}=\mathbf{A}_{a}^{T}\left(\mathbf{B}_{a}^{\dagger}\right)^{T},
$$

which shows that equation (22) and equation (26) give the same result.

4.2. XOR Using the LC Principle. The LC principle is based on projecting the experimental mode shapes onto a subset of FE modes and can, therefore, not be used for reduction. Thus, using LC to calculate the XOR is based on expanding the experimental mode shapes although the final derivation is not actual necessary. The expression for XOR equation (3) can be expressed in terms of the LC principle by inserting the expression for the expanded mode shape in matrix formulation:

$$
\mathbf{X O R}_{\text {full }}=\mathbf{P}^{T} \mathbf{B}^{T} \mathbf{M B}
$$

Due to the orthogonality conditions, the last part of equation (27) can be substituted with the identity matrix:

$$
\mathbf{X O R}_{\text {full }}=\mathbf{P}^{T} \mathbf{I}
$$

which means that the XOR corresponds to the transformation matrix:

$$
\mathbf{X O R}_{\text {full }}=\mathbf{P}^{T},
$$

where the transformation matrix consists of column vectors found individually using the procedure described with equation (18) and equation (19):

$$
\mathbf{P}=\left[\begin{array}{lllll}
\mathbf{p}_{1} & \mathbf{p}_{2} & \mathbf{p}_{3} & \ldots & \mathbf{p}_{m}
\end{array}\right] .
$$

4.3. Comparing SEREP and LC-BasedXOR. Basically, LC and SEREP use the same equations when calculating the XOR which is seen by comparing equation (22) with equation (30). There is though one very important difference between the two methods. SEREP is usually performed in one step, 
using a fixed base of FE modes. Contrary LC uses a unique base of FE modes for each experimental mode. This means that the XOR will have a different form when using the two methods. This is theoretically illustrated in Figure 1 for two models with 5 modes. SEREP will produce a square XOR with values close to 1 on the diagonal, but with values on all the elements. LC will (often) produce a rectangular XOR because of the last mode, and in these cases, the $5^{\text {th }}$ is estimated as a linear combination, for example, FE mode 3 to 6 . This also means that a number of the elements in the XOR cannot be estimated and will have the value zero.

The fact that the LC only utilizes a unique cluster of modes for each experimental mode has the advantage that noise will have much less effect on the off-diagonal element and thereby provides a more robust calculation of the XOR.

\section{Numerical Simulation}

To investigate how the LC principle performs in calculating the XOR the method was validated using numerical simulations. The primary focus of the study was to see how sensitive the method is towards noise on the experimental mode shape. When calculating the XOR, the result will depend on many different aspects, but primarily the quality of the FE model, the amount of noise on the experimental modes, and the number and placement of sensors. A probabilistic approach was there for choosing where Monte Carlo simulations were used to simulate different noise scenarios and different material properties for the FE models. For each simulation, the XOR is calculated using LC, SEREP, and Guyan.

5.1. Simulation Model. A structure consisting of three $3 \mathrm{~mm}$ steel plates was used as a case study (see Figure 2). The structure was pinned supported in all 4 corners of the bottom plate, and all connections between the plates were modelled as rigid.

An FE model of the structure was made in Nastran [22] using 377 nodes and 336 QUAD4 elements. The 6 first mode shapes were used in the analysis (see Figure 3).

Even though the structure is fairly simple, the mode shapes turn out to have rather complex forms with the structure bending and rotating in all three directions.

5.2. Perturbation of the Model. To make the simulations as realistic as possible, two versions of the FE model were made: Model A to simulate the experiment and Model B to simulate the analytical model. Three characteristics were changed to separate the experimental model from the analytical model.

5.2.1. Change of Material Properties. When making an FE model, it is almost impossible to know the exact material properties of the structure. To compensate for this, the density and E-modulus were randomly changed within predefined settings for each simulation:

$$
E_{\text {top }}=E \alpha_{\text {top }} \text { and } \rho_{\text {top }}=\rho \alpha_{\text {top }},
$$

with subscript top indicating the top plate of the structure, $E$ and $\rho$ being the original E-modulus and density, respectively, and $\alpha$ being random generated number between $1-$ $\varepsilon_{\text {top }}$ and $1+\varepsilon_{\text {top }}$, where $\varepsilon_{\text {top }}$ defines the boundaries of the size of the perturbation. Similar formulas can be written for the "bottom" and "middle" plates, with the boundaries here defined by $\varepsilon_{\text {bot }}$ and $\varepsilon_{\text {mid }}$.

5.2.2. Nonuniform Material Distribution. A test specimen will hardly ever consist of materials with uniform density and stiffness distribution. To take this into account, the mass matrix of Model A was perturbed with random generated values within a predefined threshold:

$$
\mathbf{M}_{A, \beta}=\mathbf{M}+\Delta \mathbf{M},
$$

with $\mathbf{M}_{A, \beta}$ being the perturbed mass matrix, $\mathbf{M}$ being the original mass matrix of model $\mathrm{A}$, and the elements in $\Delta \mathbf{M}$ being defined by

$$
\Delta \mathbf{M}_{i, j}=\mathbf{M}_{i, j}+\mathbf{M}_{i, j} \varepsilon_{M} \alpha,
$$

with $i$ and $j$ being the row and column number, respectively, $\varepsilon_{M}$ being the scaling factor, and $\alpha$ being random numbers between 0 and 1 . In equation (33), $\Delta \mathbf{M}$ is always forced to be symmetrical.

5.2.3. Noise on the Experimental Mode Shapes. Noise is added to Model A's mode shapes to simulate the inaccuracies caused by noise on the measured signal, noise due to identification algorithms, lack of sensor calibration etc., which affect the quality of the experimental mode shapes. The noise model is created as a vector of Gaussian random numbers scaled as a percentage of the standard deviation of the given mode shape:

$$
\mathbf{a}_{i, \beta}=\mathbf{a}_{i}+\varepsilon_{A} \boldsymbol{\beta} \sigma\left(\mathbf{a}_{i}\right),
$$

with $\mathbf{a}_{i, \beta}$ being the noised version of the $i$-th mode shape, $\varepsilon_{A}$ being the scaling factor, $\beta$ being a vector of Gaussian distributed random numbers, and $\sigma\left(\mathbf{a}_{i}\right)$ being the standard deviation of the $i$-th mode shape.

5.3. Pretest Analysis. Initial considerations were made with respect to how the active DOFs should be selected to achieve the best results. The simulations were performed using two different numbers of active DOFs: one with 15 and one with 25. The active DOFs were selected according to the effective independence method (EIM) [23] using the first 6 mode shapes as target modes. The position of the active DOFs in the two scenarios is shown in Figures 4 and 5.

Two limitations were introduced in the application of the EIM. The first being that the active DOFs should be placed perpendicular to the shell elements and the second that they could not be placed within $30 \mathrm{~mm}$ of one another. The second limitation was to prevent any clustering of the active DOFs. 

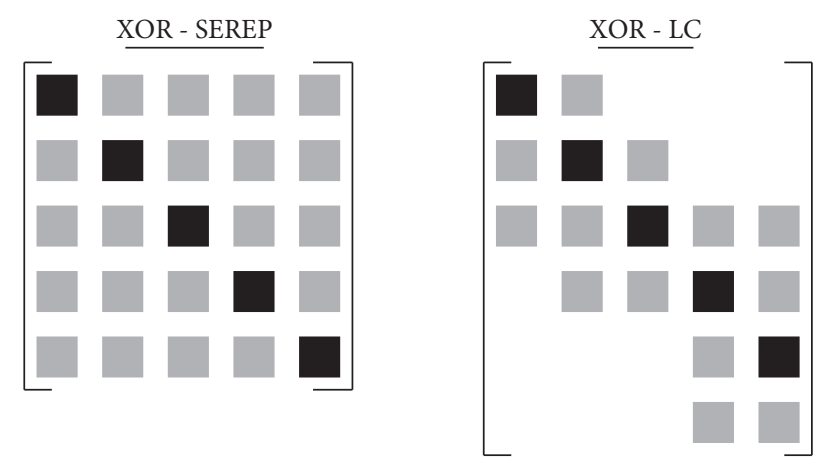

FIGURE 1: Theoretical illustration of the XOR matrix for two well-correlated models using SEREP (a) and LC (b). Black elements illustrate values close to 1 , and grey elements illustrate values close to 0.

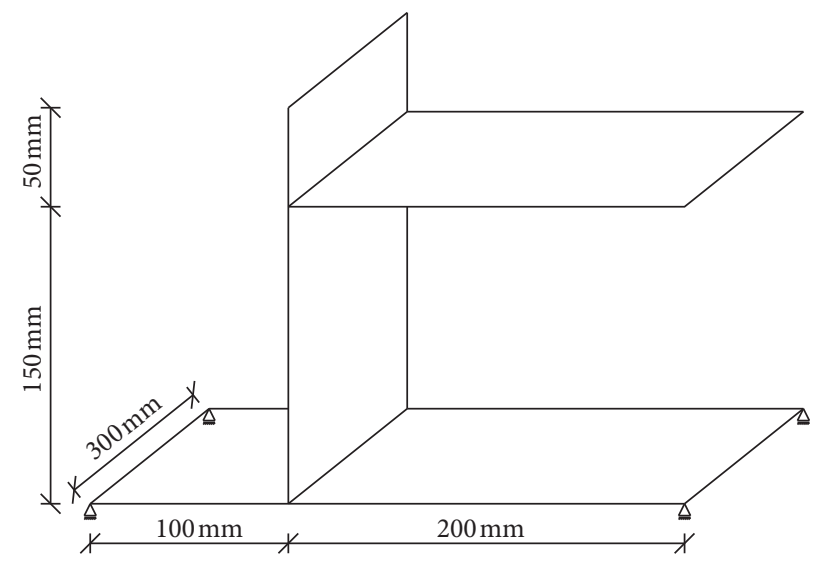

FIGURE 2: Illustration of the structure used in the numerical analysis.

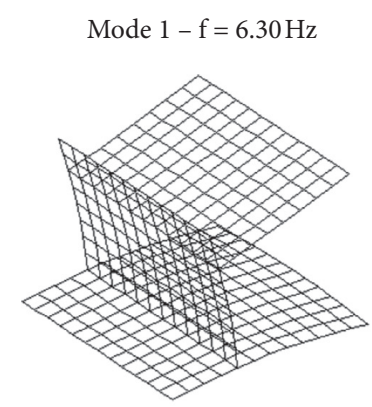

Mode $4-\mathrm{f}=16.7 \mathrm{~Hz}$

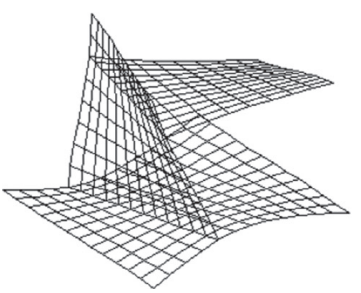

Mode $2-\mathrm{f}=13.6 \mathrm{~Hz}$

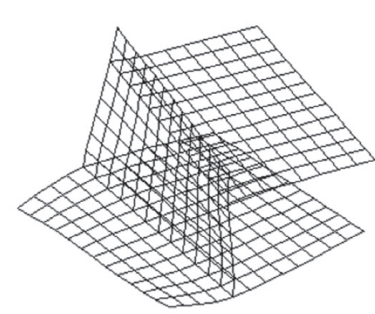

Mode $5-\mathrm{f}=20.8 \mathrm{~Hz}$

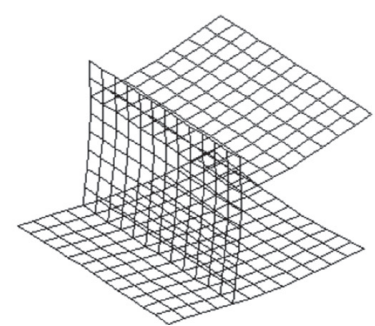

Mode $3-\mathrm{f}=14.6 \mathrm{~Hz}$

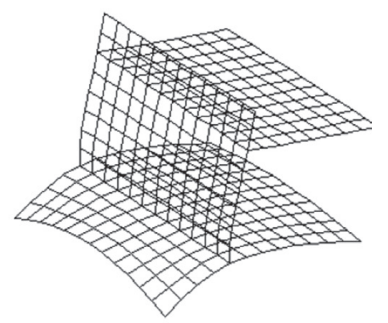

Mode $6-\mathrm{f}=36.3 \mathrm{~Hz}$

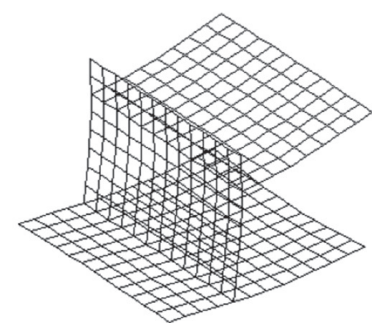

Figure 3: First 6 mode shapes from the FE model. 


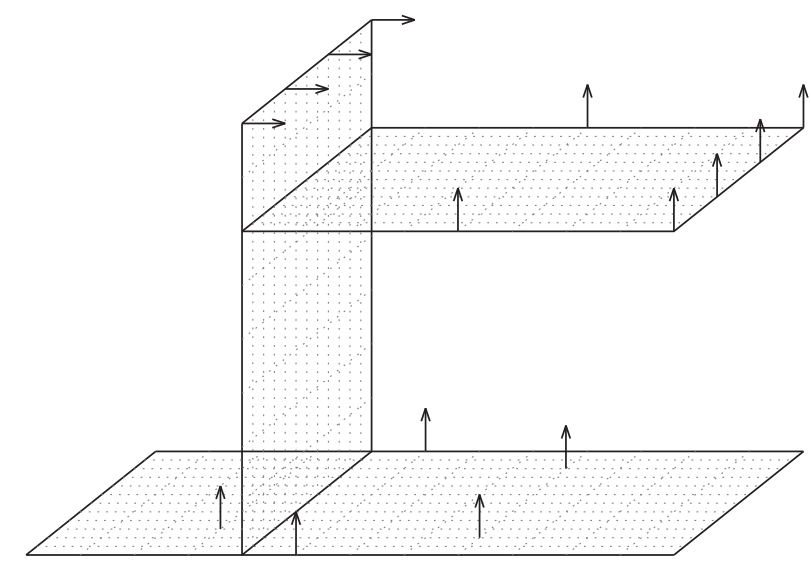

FIgURE 4: Scenario with 15 active DOFs according to the EIM.

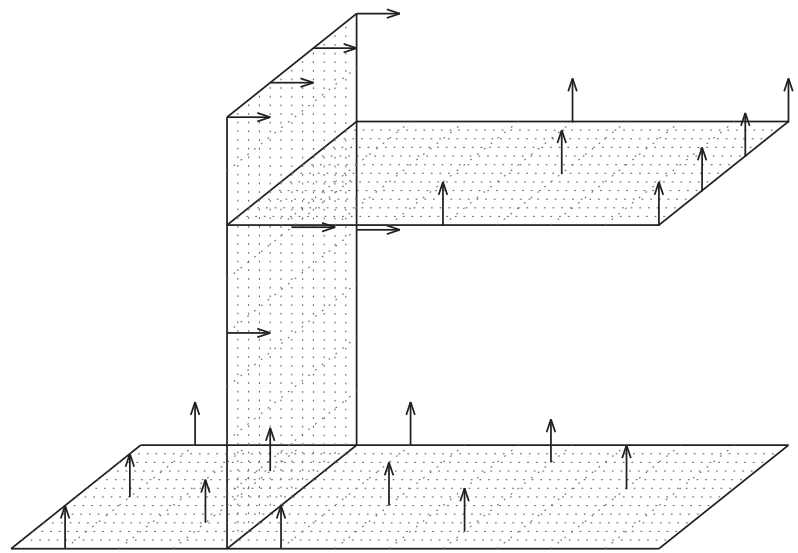

FIgURE 5: Scenario with 25 active DOFs according to the EIM.

\section{Results}

The numeric simulations were performed with two different sensor setups and with three different levels of noise on the experimental mode shapes, given a total of six different cases. For all cases, the XOR was calculated using LC, SEREP, and Guyan. Furthermore, the XOR was calculated using all the DOFs in Model A and Model B without noise on the mode shapes. This was done to see whether the exceedance of the threshold values given in equation (4) was due to actual changes in the models or due to noise. In other words, this was used as an upper measure for how well the three methods could perform.

Each of the cases consists of 30,000 simulations, where the largest off-diagonal element and the smallest diagonal element in the XOR matrices were saved for each runthrough. A detailed description of the framework for the numerical simulations is described in Appendix.

The parameters for perturbing model equations (31), (32), and (33) are described in Table 1.

For a visual presentation of the results, Kernel distribution functions were fitted to the results for each of the methods in all 6 cases. These are illustrated in Figures 6-11.
Tables 2 and 3 show the percentage of simulation outcomes which does not exceed the thresholds given in equation (2).

The first column of Tables 2 and 3 shows the results for the full system with no noise added to the mode shapes and can be seen as an upper limit for how well the three methods can perform. It shows that $100 \%$ of the minimum diagonal values are above the threshold of 0.95 , and approximately $68 \%$ of the off-diagonal values are below the threshold of 0.1 . The reason why this number varies a little in the first column of Table 2 is simply that the simulations were performed one at a time.

In the first case, 3.0\% noise was added to the mode shapes which must be considered as a low noise level and very hard to achieve in real measurements. Both LC and SEREP perform well for the off-diagonal elements, whereas Guyan only performs well in the setup with 25 sensors. This tendency is the same when looking at the diagonal terms, although SEREP here performs a little better than LC with both 15 and 25 sensors.

This picture changes when looking at the second case where $6.0 \%$ noise is added to the mode shapes. Here, LC performs considerably better for the off-diagonal elements 
TABLE 1: List of chosen parameters to perturb the reference model in the simulations.

\begin{tabular}{lcc}
\hline Parameter & Value & Description \\
\hline Top plate, $\varepsilon_{\text {top }}$ & 0.10 & The density and E-modulus varied $\pm 10 \%$ compared to the original values \\
Middle plate, $\varepsilon_{\text {mid }}$ & 0.12 & The density and E-modulus varied $\pm 12 \%$ compared to the original values \\
Bottom plate, $\varepsilon_{\text {bot }}$ & 0.08 & The density and E-modulus varied $\pm 8 \%$ compared to the original values \\
Mass matrix, $\varepsilon_{M}$ & 0.02 & The individual elements in the mass matrix varied $\pm 2 \%$ compared to the original values \\
\hline
\end{tabular}

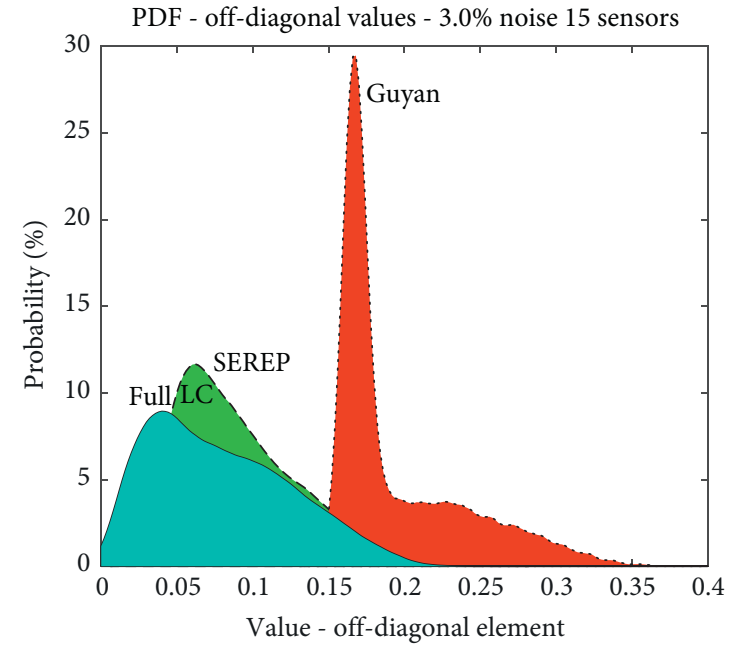

(a)

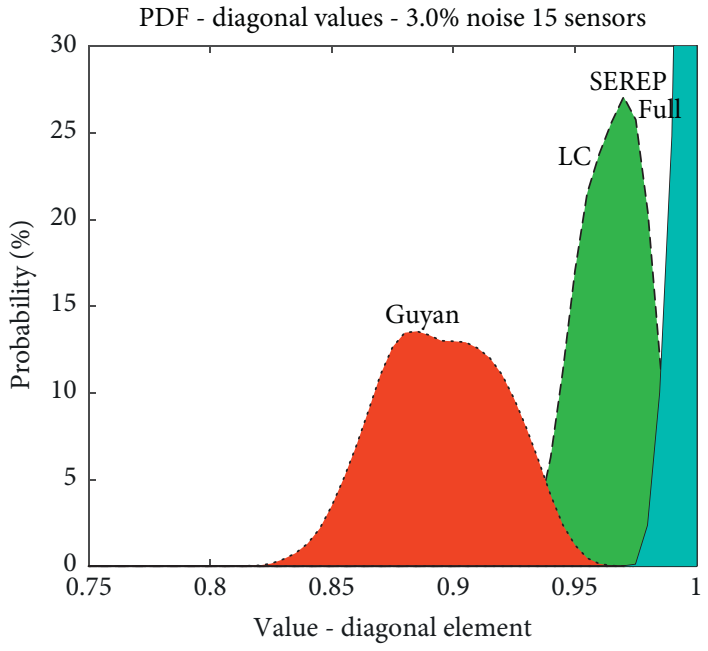

(b)

FIGURE 6: (a) PDFs of the maximal off-diagonal elements. (b) PDFs of the minimal diagonal elements. Both with 3 pct. Noise on the modes and 15 sensors.

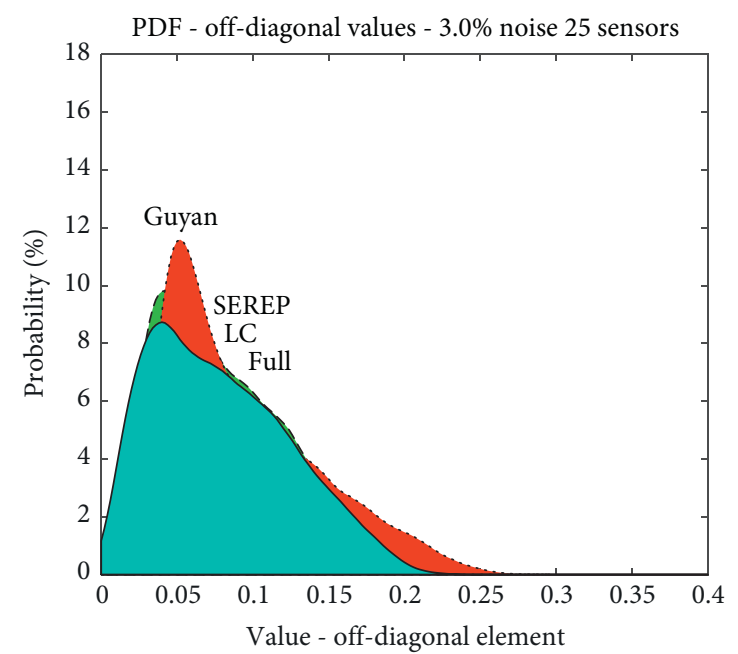

(a)

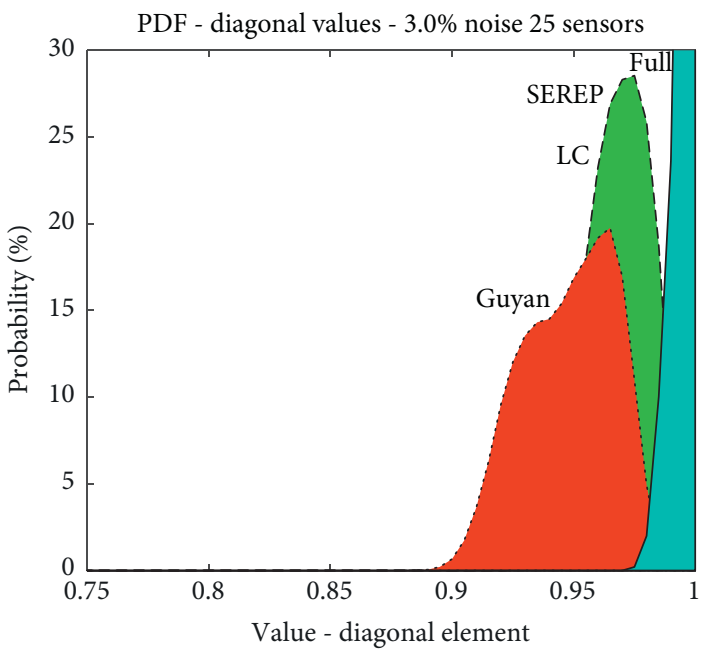

(b)

Figure 7: (a) PDFs of the maximal off-diagonal elements. (b) PDFs of the minimal diagonal elements. Both with 3 pct. Noise on the modes and 25 sensors.

in the setup with 15 sensors. Using 25 sensors, the results are more or less than the same for LC and SEREP with Guyan being a little less precise. For the diagonal terms, it is seen that the results for LC and SEREP are almost the same, with
LC having the best result using 15 sensors and SEREP having the best results using 25 sensors.

In the third case with $9.0 \%$ noise added to the mode shapes, LC still has $42 \%$ of the outcome below the given 


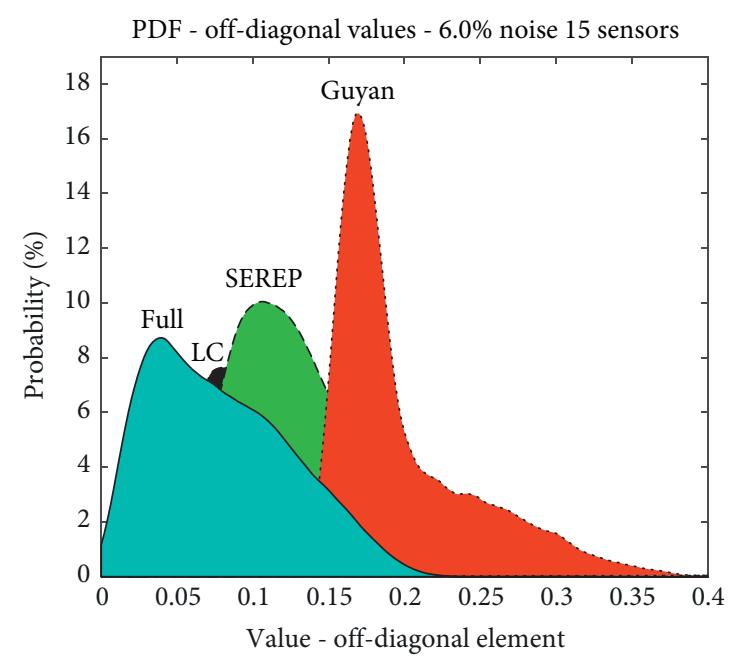

(a)

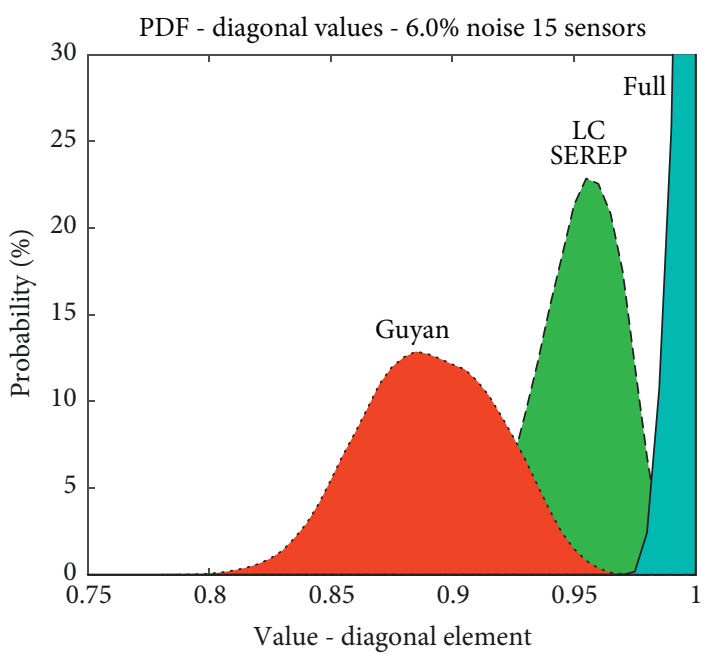

(b)

Figure 8: (a) PDFs of the maximal off-diagonal elements. (b) PDFs of the minimal diagonal elements. Both with 6 pct. Noise on the modes and 15 sensors.

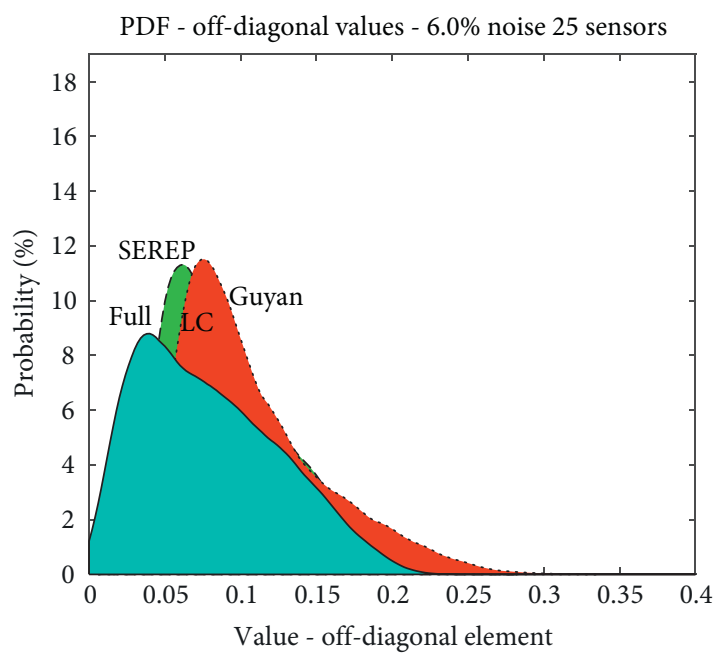

(a)

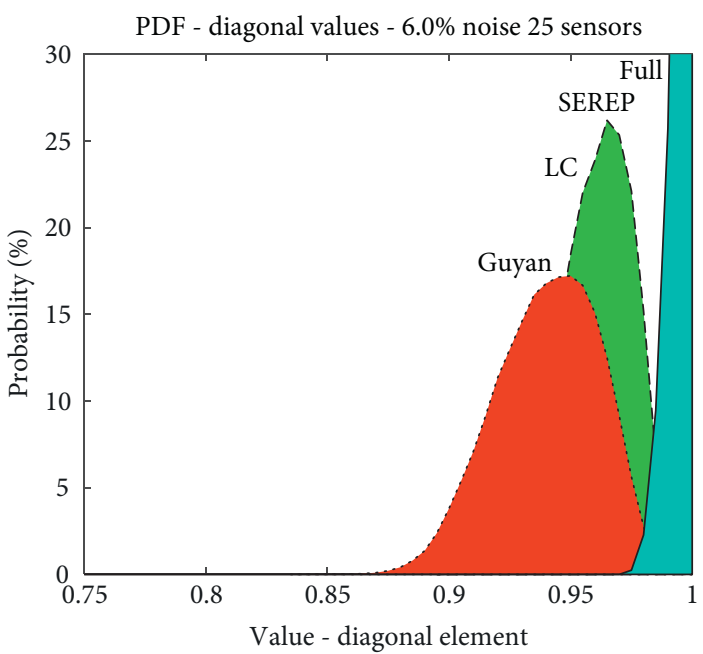

(b)

Figure 9: (a) PDFs of the maximal off-diagonal elements. (b) PDFs of the minimal diagonal elements. Both with 6 pct. Noise on the modes and 25 sensors.

threshold for the off-diagonal elements, where both SEREP and Guyan show poor results in comparison with $5 \%$ and $0 \%$, respectively. Using 25 sensors, these results improve considerably for SEREP and Guyan. The results for the diagonal terms show that LC is better using both 15 and 25 sensors, although the difference in the last case is small.

In general, all three methods loose accuracy as more noise is added to the mode shapes, which is no surprise. LC shows superior to the other methods when focusing on the off-diagonal terms. The difference is fairly small when only little noise is added to the modes but becomes more distinct in the cases with $6.0 \%$ and $9.0 \%$ noise. Furthermore, it shows that the difference in using 15 and 25 sensors is less distinct for LC than the two other methods.

For the diagonal terms, SEREP appears superior in the case of $3.0 \%$ noise. With $6.0 \%$ noise, LC and SEREP are performing with more or less same accuracy, and with $9.0 \%$, LC is superior to the other methods. 


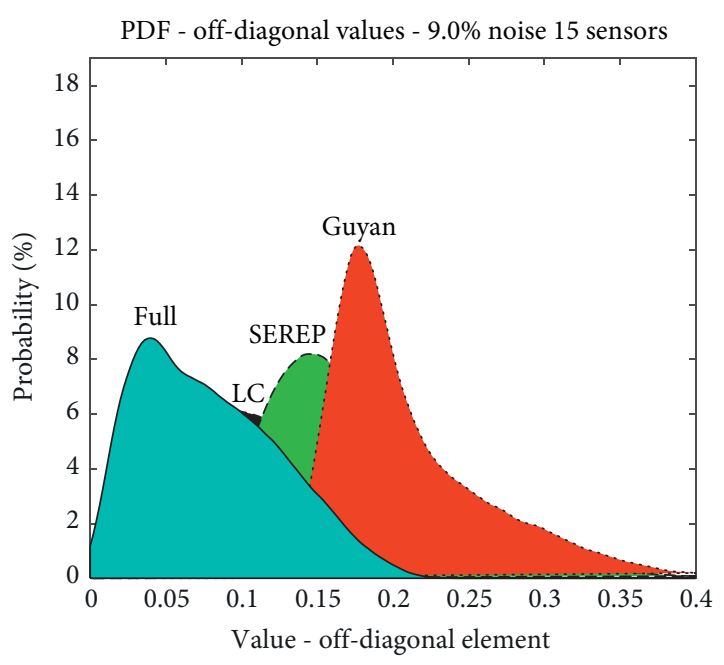

(a)

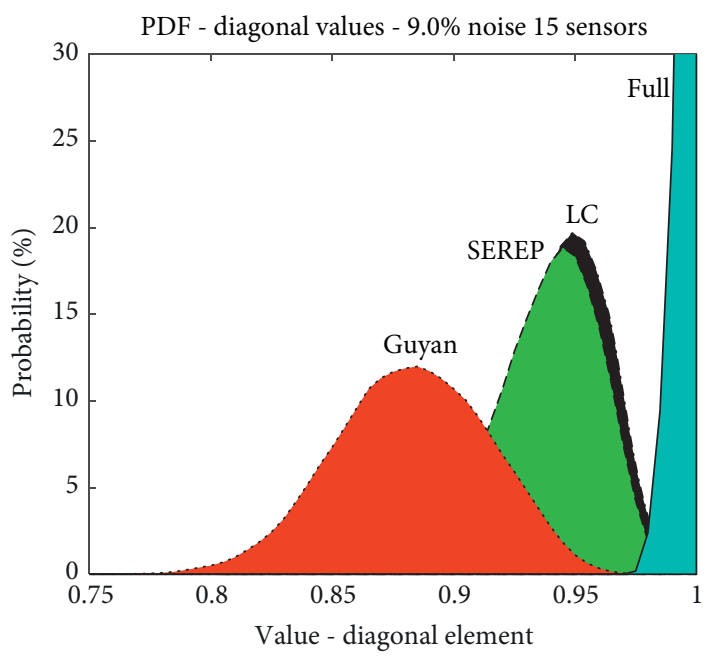

(b)

Figure 10: (a) PDFs of the maximal off-diagonal elements. (b) PDFs of the minimal diagonal elements. Both with 9 pct. Noise on the modes and 15 sensors.

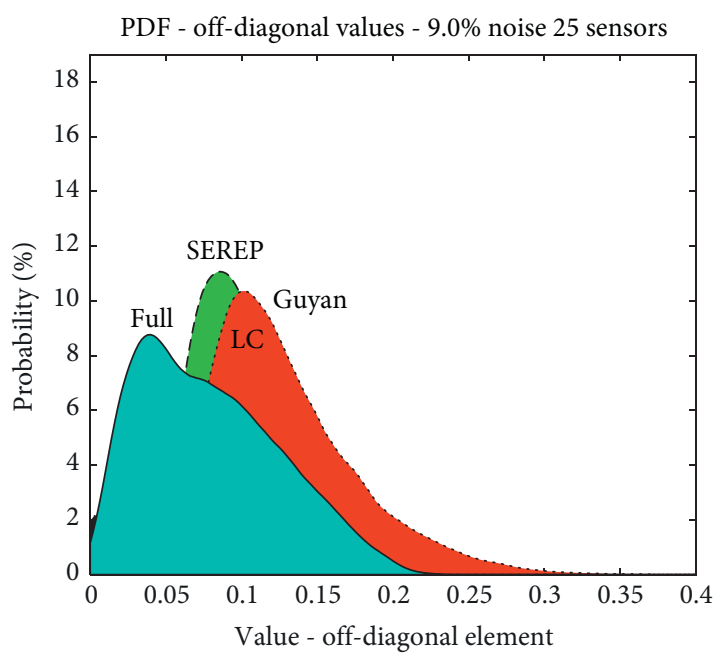

(a)

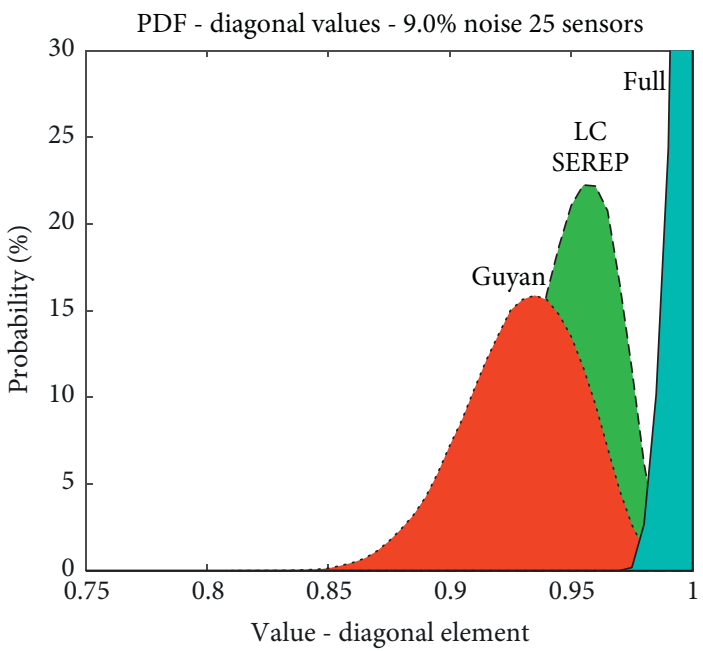

(b)

Figure 11: (a) PDFs of the maximal off-diagonal elements. (b) PDFs of the minimal diagonal elements. Both with 9 pct. Noise on the modes and 25 sensors.

TABLE 2: The percentages of the simulation results where the largest off-diagonal element in the XOR matrices stays within the threshold of 0.1 .

\begin{tabular}{lcccc}
\hline Cases of off-diagonal & Full & LC & SEREP & Guyan \\
\hline 15 sensors, 3 pct. Noise (\%): & 68.2 & 66.2 & 64.1 & 0 \\
25 sensors, 3 pct. Noise (\%): & 68.4 & 67.5 & 67.1 & 62.2 \\
15 sensors, 6 pct. Noise (\%): & 68.2 & 53.0 & 29.1 & 0 \\
25 sensors, 6 pct. Noise (\%): & 68.1 & 63.7 & 61.6 & 53.8 \\
15 sensors, 9 pct. Noise (\%): & 68.1 & 42.8 & 5.64 & 0 \\
25 sensors, 9 pct. Noise (\%): & 68.2 & 57.9 & 46.6 & 30.1 \\
\hline
\end{tabular}

TABLE 3: The percentage of simulation results where the lowest diagonal element in the XOR matrices stays above the threshold of 0.95 .

\begin{tabular}{lcccc}
\hline Cases of diagonal & Full & LC & SEREP & Guyan \\
\hline 15 sensors, 3 pct. Noise (\%): & 100 & 79.9 & 85.0 & 0.43 \\
25 sensors, 3 pct. Noise (\%): & 100 & 87.7 & 92.8 & 49.9 \\
15 sensors, 6 pct. Noise (\%): & 100 & 60.2 & 58.4 & 0.85 \\
25 sensors, 6 pct. Noise (\%): & 100 & 73.5 & 76.9 & 35.9 \\
15 sensors, 9 pct. Noise (\%): & 100 & 41.4 & 32.5 & 0.76 \\
25 sensors, 9 pct. Noise (\%): & 100 & 58.0 & 56.6 & 21.8 \\
\hline
\end{tabular}




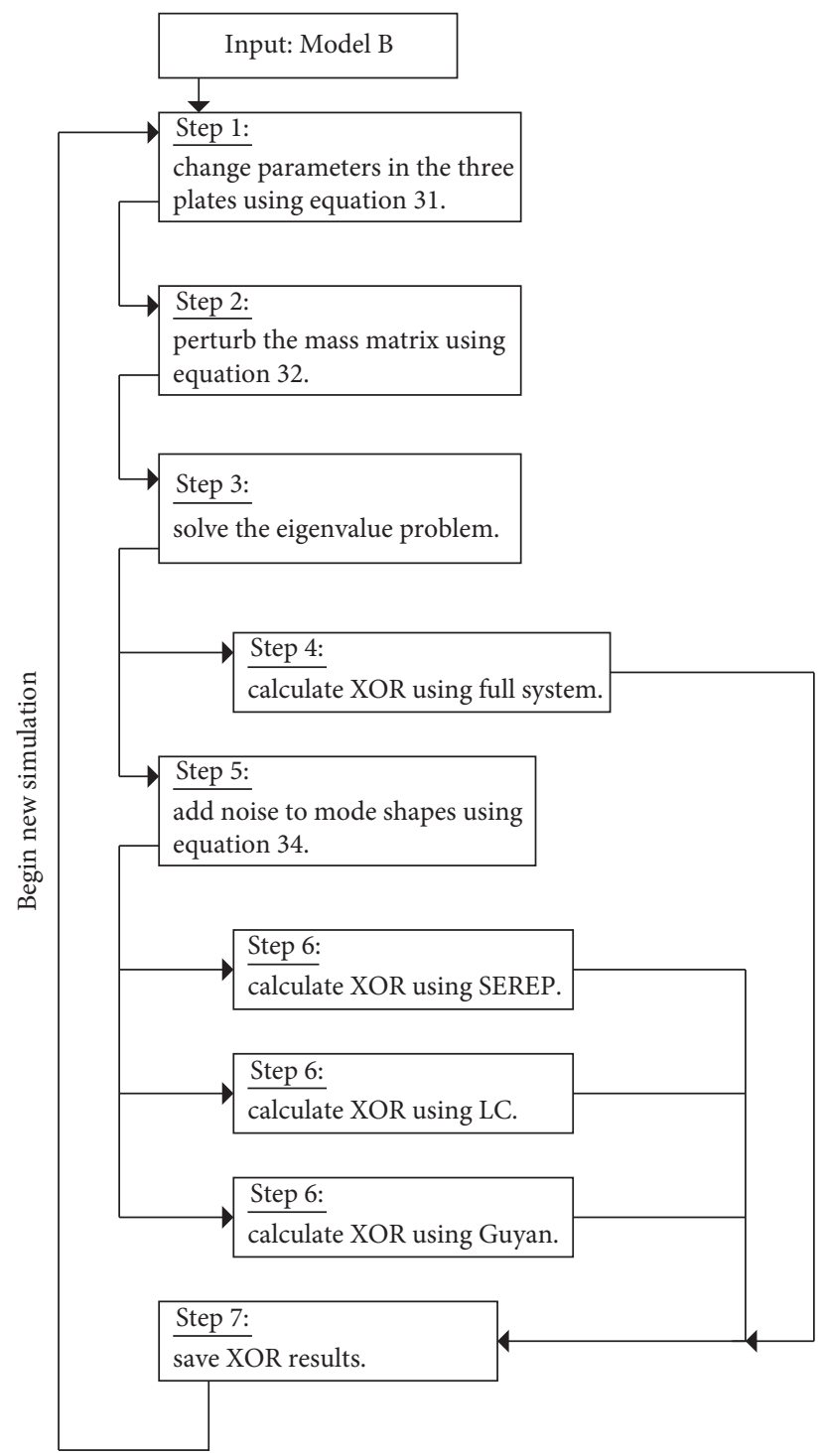

FIGURE 12: Illustration of the framework of the numerical simulations.

\section{Conclusion}

The paper presents a new method of calculating the XOR based on the principle of local correspondence. Each experimental mode shape is expressed as a unique linear combination of $\mathrm{FE}$ modes, and it is theoretically shown that the matrix describing this linear combination is equivalent to the XOR.

This new method enables a calculation of the XOR without any reduction or expansion, and without any form of mass matrix, but simply by using the experimental mode shapes and the FE mode at the active DOFs.

The method robustness towards noise on the experimental mode shape is validated in a case study using a probabilistic approach. Simulations are performed with three different levels of noise, two different sensor locations, and material properties changing stochastically. The results are compared with Guyan and SEREP. When focusing on the off-diagonal terms of the XOR, LC shows superior to the other methods for all three noise scenarios. For the diagonal terms, SEREP shows superior when only a little noise is added, whereas LC shows better results in the cases with higher noise level.

\section{Appendix}

This appendix will shortly describe how the numerical simulations were performed with reference to the theory presented in the main article. Each simulation consists of 7 steps, using Model B as input. The main purpose of the simulations was to create two models which represented an experimental model (Model A) and an analytical model (Model B) and use three different methods to calculate the XOR.

This was done using Model B as input, creating Model A by changing the material properties, and perturbing the mass matrix. Further noise was added to the mode shapes from Model A, to simulate noise from measurements and identification algorithms. 
Step 1. The density and E-modulus is randomly changed individually for the three plates, using equation (31). For the top plate, changes were in the range of $\pm 10 \%$, for the middle plate $\pm 12 \%$ and for the bottom plate $\pm 8 \%$.

Step 2. The mass matrix is perturbed with Gaussian white noise according to equation (32) and equation (33). Each element was randomly changed within $\pm 2 \%$ of its original value. The changes were applied, so the new mass matrix was symmetrical.

Step 3. By using the new mass and stiffness matrices created in Step 1 and Step 2, the mode shapes and frequencies for Model A are found by solving the eigenvalue problem.

Step 4. The XOR is calculated for the full system using all known DOFs in the two models.

Step 5. Noise is added to the mode shape from Model A, using equation (34). The noise consists of Gaussian-generated vectors scaled to the standard deviation of the given mode shapes. Different noise levels are applied.

Step 6. The XOR is calculated using Guyan, SEREP, and LC. The first 6 modes are used as target modes in the simulations.

Step 7. For each of the 4 XOR matrices, the smallest diagonal element and the largest off-diagonal element are saved.

The procedure is illustrated in Figure 12.

\section{Data Availability}

Simulations used in the paper are available upon request from the corresponding author.

\section{Conflicts of Interest}

The authors declare that they have no conflicts of interest.

\section{Acknowledgments}

The economic support given by the Cajastur Fellowship Programme through the project SV-11-CAJASTURFP-4 and the Spanish Ministry of Economy and Competitiveness through the project BIA2011-28380-C02-01 is gratefully appreciated.

\section{References}

[1] R. J. Allemang, "The modal assurance criterion-twenty years of use and abuse," Sound and Vibration Magazine, vol. 37, no. 8, pp. 14-21, 2003.

[2] T. K. Hasselman, R. N. Coppolino, and D. C. Zimmerman, "Criteria for modeling accuracy: A state-of-the-practice survey," in Proceedings of the 18th IMAC Conference, pp. 335-341, San Antonio, TX, USA, February 2000.

[3] NASA, NASA-STD-5002, Loads Analyses of Spacecraft and Payloads, NASA, Washington, DC, USA, 1996.

[4] ECSS-E-ST-32-11C, Modal Survey Assessment, European Space Agency, Paris, France, 2008.
[5] R. J. Guyan, "Reduction of stiffness and mass matrices," AIAA Journal, vol. 3, no. 2, p. 380, 1965.

[6] R. L. Kidder, "Reduction of structural frequency equations," AIAA Journal, vol. 11, no. 6, p. 892, 1973.

[7] O'Callahan, "A procedure for an improved reduced system (IRS) model," in Proceedings of the Seventh International Modal Analysis Conference, Las Vegas, NV, USA, February 1989.

[8] J. C. O'Callahan, P. A. Avitabile, and R. Riemer, "System equivalent reduction expansion process (SEREP)," in Proceedings of the Seventh International Modal Analysis Conference, Las Vegas, NV, USA, February 1989.

[9] D. C. Kammer, "Test-analysis model development using an exact modal reduction," International Journal of Analytical and Experimental Modal Analysis, vol. 2, pp. 174-179, 1987.

[10] K. F. Martin and K. H. Ghlaim, "Non orthogonality arising from repeated roots," in Proceedings of the 5th International Modal Analysis Conference, pp. 1331-1337, London UK, April 1987.

[11] Y. Matsumura, N. Tsujiuchi, and T. Koizumi, "Cross orthogonality check using the general description of projection," in Proceedings of the 16th International Modal Analysis Conference, pp. 1395-1400, Santa Barbara, CA, USA, February 1998.

[12] Y. Matsumura, "Uncertainty in cross orthogonality checks," JSME International Journal Series C, vol. 46, no. 2, pp. 692698, 2003.

[13] E. J. Bergman, M. S. Allen, D. C. Kammer, and R. L. Mayes, "Probabilistic investigation of sensitivities of advanced testanalyses-model correlation," Journal of Sound and Vibration, vol. 329, no. 13, pp. 2516-2531, 2010.

[14] G. S. Aglietti, S. J. I. Walker, and A. Kiley, "On the use of SEREP for satelite FEM validation," Engineering Computations, vol. 29, no. 6, pp. 580-595, 2012.

[15] K. K. Sairajan and G. S. Aglietti, "Robustness of system equivalent reduction expansion process on spacecraft structure model validation," AIAA Journal, vol. 50, no. No. 11, pp. 2376-2388, 2012.

[16] S. R. Aglietti, S. J. I. Walker, and A. Kiley, Robustness of the Orthogonality Checks on a Satellite FEM Using a SEREP Test Analysis Model, in Proceedings of ISMA 2010, pp. 20-22, Leuven, Belgium, September 2010.

[17] R. Brincker, A. Skafte, M. López-Aenlle, A. Sestieri, W. D'Ambrogio, and A. Canteli, "A local correspondence principle for mode shapes in structural dynamics," Mechanical Systems and Signal Processing, vol. 45, no. 1, pp. 91-104, 2014.

[18] R. B. Nelson, "Simplified calculations of eigenvector derivatives," AIAA Journal, vol. 14, no. 9, pp. 1201-1205, 1976.

[19] W. Heylen, S. Lammens, and P. Sas, Modal Analysis Theory and Testing, Katholieke Universiteit Leuven, Leuven, Belgium, 1997.

[20] A. T. Johansson and T. J. S. Abrahamsson, "Selecting appropriate analytical mode basis for SEREP-expansion of experimental modes," Conference Proceedings of the Society for Experimental Mechanics Series, vol. 3, pp. 1-15, 2011.

[21] P. Avitabile and J. C. O'Callahan, "Mass and stiffness orthogonality checks without a mass or stiffness matrix," in Proceedings of the 13th International Modal Analysis Conference, Nashville, TN, USA, February 1995.

[22] Patran/Nastran, 2012, MSC Software.

[23] D. C. Kammer, "Sensor set expansion for modal vibration testing," Mechanical Systems and Signal Processing, vol. 19, no. 4, pp. 700-713, 2005. 